\title{
BMJ Open COVID-19 welbeing study: a protocol examining perceived coercion and psychological well-being during the COVID-19 pandemic by means of an online survey, asynchronous virtual focus groups and individual interviews
}

\author{
Veronica Ranieri (10 , ${ }^{1,2}$ Andrea Sem Stoltenberg, ${ }^{1}$ Elena Pizzo, ${ }^{3}$ Chiara Montaldo, ${ }^{4}$ \\ Emanuele Bizzi, ${ }^{5}$ Sarah Edwards, ${ }^{2}$ Sunjeev Kamboj ${ }^{6}$
}

To cite: Ranieri V, Sem Stoltenberg A, Pizzo E, et al. COVID-19 welbeing study: a protocol examining perceived coercion and psychological well-being during the COVID-19 pandemic by means of an online survey, asynchronous virtual focus groups and individual interviews. BMJ Open 2021;11:e043418. doi:10.1136/ bmjopen-2020-043418

- Prepublication history and additional material for this paper are available online. To view these files, please visit the journal online (http://dx.doi. org/10.1136/bmjopen-2020043418).

SE and SK are joint senior authors.

Received 03 August 2020 Revised 17 October 2020 Accepted 12 January 2021

Check for updates

(c) Author(s) (or their employer(s)) 2021. Re-use permitted under CC BY-NC. No commercial re-use. See rights and permissions. Published by BMJ.

For numbered affiliations see end of article.

Correspondence to Dr Veronica Ranieri; v.ranieri@ucl.ac.uk

\section{ABSTRACT}

Introduction The COVID-19 pandemic has resulted in many countries applying restrictive measures, such as lockdown, to contain and prevent further spread. The psychological impact of lockdown and working as a healthcare worker on the frontline has been chronicled in studies pertaining to previous infectious disease pandemics that have reported the presence of depressive symptoms, anxiety, insomnia, and post-traumatic stress symptoms. Potentially linked to psychological well-being and not yet studied is the possibility that lockdown and working on the frontline of the pandemic are associated with perceptions of coercion.

Methods and analysis The present study aimed to examine perceived coercion in those who have experienced COVID-19-related lockdown and/or worked as a frontline healthcare worker across three European countries. It aimed to describe how such perceptions may impact on psychological well-being, coping and posttraumatic growth. It will employ an explanatory mixedmethods research methodology consisting of an online survey and online asynchronous virtual focus groups (AVFGs) and individual interviews. $\chi^{2}$ tests and analyses of variance will be used to examine whether participants from different countries differ according to demographic factors, whether there are differences between cohorts on perceived coercion, depression, anxiety and post-traumatic growth scores. The relationship between coercion and symptoms of distress will be assessed using multiple regression. Both the AVFGs and the narrative interviews will be analysed using thematic narrative analysis.

Ethics and dissemination The study has been approved by University College London's Research Ethics Committee under Project ID Number 7335/004. Results will be disseminated by means of peer-reviewed publications and at national and/or international conferences.

\section{INTRODUCTION}

COVID-19 is an infectious disease caused by a novel betacoronavirus believed to originate
Strengths and limitations of this study

- This study will use online quantitative and qualitative research methods to reach both the general population and key workers affected by the COVID-19 lockdown.

- It will adopt the use of innovative research methods, such as asynchronous virtual focus groups, to allow participants to describe experiences of lockdown and working in frontline roles during the pandemic, and to communicate with other participants openly and flexibly.

- The methods allow for replicability and cultural adaptation across different countries.

- Involvement in this study is dependent on literacy, tech literacy and internet access.

in Wuhan, China. ${ }^{1-3}$ Early indications of the presence of this virus emerged in December 2019, when several individuals displayed clinical presentations akin to viral pneumonia. ${ }^{2}$ Although our knowledge of the virus is still accumulating, severe cases of infection may lead to serious health complications and death. ${ }^{2}$ As of 11 March 2020, the WHO declared the disease a pandemic. In addition to this declaration, individuals and governments were advised to take precautionary and restrictive measures to reduce virus transmission, such as social distancing, self-isolation, quarantine and lockdown, depending on prevalence and health service capacity within each country. ${ }^{4}$

The psychological impact of restrictive measures and epidemics has received some attention in the mental health literature, with studies reporting the presence of depressive symptoms, anxiety and insomnia in those who 
have experienced quarantine and those who have worked as healthcare workers during epidemics. ${ }^{5-13}$ Such distress, as well as its possible impact on suicidality, is expected as a result of restrictive practices linked to the COVID-19 pandemic, with researchers and clinicians worldwide commenting on the effect of secondary stressors associated with both the lockdown and the threat of infection on the general population's mental health. ${ }^{14-19}$ In healthcare workers, it has been linked to a number of COVIDrelated work stressors, including increased workloads, unfamiliar tasks, being at increased risk of infection and fears of infecting others. ${ }^{12} 20-23$

Linked to psychological well-being and not yet studied is the potential for these experiences to give rise to perceptions of coercion, both in those living under lockdown and those providing frontline healthcare. Such perceptions arise from experiencing a dearth of choice, freedom, influence or control with regard to one's situation. ${ }^{24} 25$ These are most commonly reported when individuals experience a situation that they feel is forced on them without justification, and where they feel excluded from the decision-making process or do not have an opportunity to express their viewpoint. ${ }^{26}$ Such perceptions have been commonly reported in the mental health literature, particularly in relation to restrictive measures such as involuntary admissions to hospital. which, in ways, may be comparable in populations where restrictions were severe and individuals were legally enforced to stay at home. ${ }^{27}$ In mental health settings, higher levels of perceived coercion are indicative of a poorer prognosis. ${ }^{28}$ It is, however, unclear as to whether a pandemic-related lockdown will give rise to similar perceptions of coercion. Understanding such perceptions is critical if governments are to secure the cooperation of their citizens in enacting mitigation (lockdown) efforts, and the cooperation and mental well-being of frontline workers, in future scenarios.

In light of the aforementioned research, the present study primarily examines perceived coercion in those who have experienced COVID-19-related lockdown and/or frontline roles across three European countries with different demographic characteristics and healthcare systems, and which enacted differentially stringent mitigation approaches. In healthcare workers, it examines how perceptions of coercion and pressures may be related to perceived risk of infection and COVID-19related stressors in the workplace. Using the transactional theory of stress and coping, it aimed to describe how such perceptions may impact on psychological well-being, coping and post-traumatic growth, ${ }^{29}$ and, in healthcare workers, on professional quality of life. Finally, we will also preliminarily investigate whether there is an increase in healthcare resource usage (in the general population sample), as a coping strategy, and whether this is linked to the aforementioned constructs.

\section{Key research questions}

Our research questions are the following:
1. What are the experiences of those who have been under lockdown or have been working as healthcare workers during the COVID-19 pandemic? How have these experiences impacted on their psychological well-being?

2. To what extent does the general population perceive the lockdown as coercive, pressured and procedurally just? To what extent do healthcare workers perceive having to work on the frontline with patients with COVID-19 as coercive? Do these perceptions change over time?

3. Are perceptions of coercion associated with psychological distress, after controlling for demographic and background factors?

4. Do perceptions of coercion and psychological distress in the general population and among key workers vary across affected countries after controlling for demographic and background factors?

5. What practical recommendations can we highlight to policy-makers and healthcare management about how to improve the psychological support provided to both the general population and health workers across affected countries?

\section{METHODS AND ANALYSIS}

This study will employ an explanatory mixed-methods research methodology consisting of (1) an online survey and (2) online asynchronous virtual focus groups (AVFGs) or individual interviews.

\section{Sample}

Online survey

Participants will consist of individuals who have experienced governmental lockdown and/or who are key workers during the coronavirus pandemic. Participants will be recruited from the UK, Italy and Norway with the potential to be extended to other countries. Individuals aged $\geq 18$ years and who have experienced governmental lockdown and/or are key workers working on the frontline in the UK, Italy or Norway will be invited to participate. We aimed to recruit 2000 individuals who experienced lockdown per country, as per MartínezMesa et $a l$ and Maxwell. ${ }^{3031}$ Participants will be recruited using social media platforms such as Twitter, Facebook and Instagram. Advertisements for the study will also be cascaded via email and other social media messaging technology such as WhatsApp.

\section{AVFGs and individual interviews}

A sample of individuals from the quantitative study will be invited to participate in either an AVFG or individual interview, depending on preference and availability. A total of six to nine AVFGs will be conducted simultaneously, with two to three AVFGs/country. AVFGs will be limited to 6-10 participants/group. Purposive sampling will be used to select individuals according to their age, gender and geographical location, or other distinctive 
factors where possible, so that each group consists of participants from a diverse range of backgrounds.

\section{Setting}

The study will be completed entirely online using a general data protection regulation (GDPR)-compliant data collection tool (ISO 27001 certified). AVFG participants will be asked to log onto a virtual learning environment $^{32-34}$ that provides a secure and confidential space for research participants to write and interact with each other. Individual interviews will take place by telephone or using online conferencing software.

\section{Procedure}

\section{Online survey}

Advertisements will be posted and shared on social media. Clicking on the study's link will direct participants to the survey's home page from which they can be directed to the study information page. Should they wish to participate after reading the study information, they will provide informed consent online and proceed to the online survey. After completing the survey, participants will be invited to take part in a shorter follow-up survey within a period of 3 months and/or further qualitative research. Those who wish to take part will be asked to enter their email address.

\section{AVFGs or individual interviews}

AVFG participants will be registered onto a virtual learning environment $^{32} 33$ under a chosen alias (to preserve anonymity). Then they will familiarise themselves with the platform and documents listed as 'essential information', which include study information, addition consent form, researcher contact details and support, and netiquette guidelines. Once all participants provide consent, the first focus group question will appear as a discussion topic. Participants will be asked to engage in discussion about a different question (outlined as follows) each week for 3 weeks. Participants will be able to post as often as they wish each week, both in response to the question and in reaction to other participants' responses. Participants will receive an email notification for each new question posted. Discussion boards will be moderated two times per day by the researchers to monitor the content of what is posted and to delete if offensive, and to probe and clarify participants' responses.

Should participants prefer to take part in an interview rather than an AVFG, the researcher will email them a copy of the information sheet and consent form and arrange a time for interview. All interviews will be audio-recorded and then transcribed. The interviews are expected to last up to 1 hour. All identifiable data will be stored on Data Safe Haven, in accordance with University College London (UCL) policy.

\section{Instruments}

Online survey

All participants will complete a questionnaire constructed by the researchers that asks individuals about general demographic details and their clinical background at baseline. Participants will also complete the following measures at baseline and within a 3-month follow-up period: (1) an adapted version of the MacArthur Admission Experience Survey ${ }^{24}$ to measure the extent to which individuals perceive their circumstances as coerced, pressured and procedurally just in the general population and a Perceived Coercion Scale for Healthcare Workers constructed by researcher AS, (2) the Depression, Anxiety and Stress Scale ${ }^{35}$ to indicate the presence of depression, anxiety, stress and post-traumatic growth; (3) the Post-traumatic Growth Inventory-Short Form ${ }^{36}$ to assess whether individuals experience positive outcomes relating to the COVID-19 pandemic; and (4) the brief $\mathrm{COPE}^{37}$ to observe individuals' methods of coping within the context of the COVID-19 pandemic. Healthcare workers will also be asked to complete an adapted version of the Perceived SARS Related Risk Scale ${ }^{38}$ and the Professional Quality of Life measure. ${ }^{39}$ The adapted versions of the scales may undergo minor modification after piloting. A copy of the survey can be accessed online (www.thec ovid19wellbeingstudy.org). A copy of the survey questions is attached as an online supplemental file with this article.

\section{AVFGs}

Members of the general population taking part in the AVFGs will be asked to answer the following questions and to engage with other participants' responses:

Week 1: Can you tell us about how you felt when you were first told to stay home during the coronavirus pandemic? Looking back on the lockdown, have your feelings changed towards it? If yes, in what way? Did you agree or disagree with the governmental lockdown? In what way? How do you feel about the lockdown being lifted? What are your feelings regarding a future potential lockdown?

Week 2: Did you self-isolate prior to the lockdown or did you remain home solely because the lockdown came in? For example, did you first experience forced isolation due to confirmation of illness? Did you first practice self-isolation due to you or someone in your household being symptomatic, or having a condition that places you at a greater health risk? Or was your first experience of isolation a result of governmental lockdown? Did you feel you had any control over your isolation? Was it something you chose initially or something you felt was forced on you? How did this make you feel?

Week 3: What has been the impact of the lockdown on your psychological well-being? Have you, someone in your household or someone you know experienced an onset of coronavirus-type symptoms? How did this impact you emotionally? What support do you feel you need for your emotional well-being? What have you done so far to try to stay well?

Healthcare workers will be asked to answer and engage with other participants' responses for the following questions: 
Week 1: Can you tell us about how you felt when you first started working with patients confirmed with or suspected of having COVID-19? Were you asked or told to work with this patient group? Did you volunteer to work with this patient group? Did you feel that working with this patient group is part of your work role, in spite of potential risk? How have your feelings changed about working with this patient group between then and now, if at all?

Week 2: Under what circumstances did you work with patients confirmed with or suspected of having COVID-19? Are you redeployed or did you remain in your workplace? Did you feel that you were adequately physically protected? Did you feel adequately supported within your workplace? Did you feel your needs were considered? Did you encounter barriers to accessing protection or support within your workplace? In what way?

Week 3: What has been the impact of working as a key worker with individuals with COVID-19 on your psychological well-being? What supports do you feel you need for your emotional well-being? Did you seek psychological support, and from whom? What have you done so far to try to stay psychologically well?

\section{Individual interviews}

Semistructured interviews will be adopted to enable the researchers to capture detailed insights about each individual's personal experiences and perceptions and the context in which these occur. These interviews will focus on individuals' perceptions regarding lockdown (inclusive of perceived coercion, pressures, and procedural justice), their experiences of isolation and their psychological well-being. Individuals who identify themselves as healthcare workers in the online survey may be invited to interview depending on their availability. These interviews will be slightly tailored to focus on clinicians' experiences and perceptions of working during the coronavirus pandemic (inclusive of perceived coercion, pressures and procedural justice), and their psychological well-being.

\section{Analysis \\ Online survey}

All numerical data will be inputted into a statistical analysis software programme (SPSS) version 26. The data will be tested for normality by conducting quartile-quartile plots and Kolmogorov-Smirnov tests for each measure (and subscale, where applicable). Following the aforementioned initial management of the data, we will describe demographic and background characteristics according to country and respondent type. A multiple regression model will be used with perceived coercion as the main predictor and measures of psychological distress (ie, depression, anxiety and post-traumatic stress) as the main outcome variables. The secondary outcome variables will include measures of adaptability (ie, coping and post-traumatic growth) and demographic (ie, age, gender, ethnicity and education level) and background variables (ie, job role and work environment) as covariates. In healthcare workers, COVID-19-related stressors and perceptions of COVID-related risk will also be analysed as possible predictors, mediators and/or moderators. Further exploratory analysis may be conducted to assess for interaction between variables. Analyses of variance will be used to examine whether there are differences between UK, Italian and Norwegian cohorts on perceived coercion, stress, depression, anxiety, trauma scores and professional quality of life.

\section{AVFGs and individual interviews}

The qualitative substudy will employ a phenomenological approach as its focus is on understanding the subjective experiences of individual participants and the meaning that participants attribute to these experiences. It will also employ an interpretative framework, using the stresscoping paradigm, whereby the focus of our questions and analysis will be on creating a picture of what influences individuals' appraisals of perceived coercion, pressures and procedural justice and how these appraisals influence and are influenced by coping. Focus group data will be downloaded directly as text from the virtual learning environment platform. Interviews will be audio-recorded and transcribed. Computer Assisted Qualitative Data Analysis Software, such as NVivo, will be used to code the transcripts. ${ }^{40}$ Text scripts and transcripts will be analysed, adapting Braun and Clarke's thematic analysis. ${ }^{41}$ This will involve both sequencing events and experiences, and grouping commonalities and experiences within chronological sequences, and between the two types of participants. Themes and narratives will be explored by two researchers with one principally analysing the data and the second overseeing the emerging themes. Any disagreements will be discussed and resolved within the wider research team. Concepts from the narratives will be both derived inductively from the data and applied deductively to our theoretical framework.

\section{Patient and public involvement}

No patient involvement. The background questionnaire was co-constructed with the authors who are healthcare workers. The questionnaire was also piloted with healthcare professionals who gave feedback on it.

\section{ETHICS AND DISSEMINATION}

This study received approval by UCL's Research Ethics Committee as application 7335/004.

\section{Informed consent}

Individuals who click on the study's advertisement on social media will be brought to the survey's home page. Should they wish to proceed, they will be brought to an information page detailing the purpose of the study, how their confidentiality and anonymity will be preserved and how their data will be treated. Should individuals wish to participate after reading the information page, they will 
be asked to provide informed consent online. At the end of the survey, participants will be asked if they would like to take part in a follow-up assessment and/or an online focus group or interview. Those who wish to take part will be asked to enter their email address.

Participants who provide their email address and show a preference to take part in an online group will be registered onto a virtual learning environment (ie, Blackboard) under an alias chosen by them in order to preserve their anonymity. Once registered, they will be asked to $\log$ in and familiarise themselves with both the platform and all documents listed as 'essential information' within it. Essential information will include the study's information sheet and separate consent form, contact details for the researchers and support, and netiquette guidelines. Once all participants provide consent online, the first focus group question will appear as a discussion topic. Should participants prefer to take part in an interview, the researcher will email them a copy of the information sheet and consent form and arrange a time for interview.

\section{Data protection and confidentiality}

An online data collection tool that is GDPR compliant and certified to ISO 27001 standard will be used for the online survey. Participants will be given the opportunity to choose their own pseudonym for the AVFGs to help preserve their anonymity. All data collected during the course of the research will be kept strictly confidential. All identifiable information will be stored on UCL's Data Safe Haven, a GDPR-compliant, encrypted system for the duration of the study. Audio recordings will be kept on Data Safe Haven until these are transcribed into an anonymous format. Non-identifiable data will be stored on a password-protected, encrypted drive on a UCL desktop for a period of 5 years.

\section{Dissemination}

Participants will not be identifiable in any ensuing reports or publications. Results will be disseminated by means of peer-reviewed publications and at national and/or international conferences. We will aim to publish the findings in an open-access journal to make the study's findings accessible to the general population. A summary of the findings will be shared on our website (thecovid19wellbe ingstudy.org)

\section{Author affiliations}

${ }^{1}$ Psychology and Language Sciences, University College London, London, UK ${ }^{2}$ Science and Technology Studies, University College London, London, UK ${ }^{3}$ Applied Health Research, University College London, London, UK

${ }^{4}$ Ambulatorio di Medicina Tropicale e del Viaggiatore, Istituto Nazionale per le Malattie Infettive Lazzaro Spallanzani, Roma, Lazio, Italy

${ }^{5}$ Dipartimento di Medicina Interna, San Pietro Fatebenefratelli Hospital, Rome, UK ${ }^{6}$ Psychology, University College London, London, UK

\section{Twitter Chiara Montaldo @montaldo_chiara}

Contributors VR and AS constructed the initial protocol. VR and ASS drafted this protocol article. SK contributed to the quantitative and psychological designs of the study. SE contributed to the research ethics design of the study. EB and CM contributed to the design of the healthcare professional questions. EP constructed the health economics questions. All authors contributed to and reviewed the present protocol.

Funding SK is supported by the Sir Bobby Charlton Foundation. SE is funded by EDCTP PANDORA-ID NET, UK NIHR UCLH/UCL Biomedical Research Centre and the Medical Research Council. EP was funded by the UK National Institute for Health Research (NIHR) Applied Research Collaboration North Thames at Barts Health NHS Trust. The views expressed are those of the authors and not necessarily those of the NHS, the NIHR or the Department of Health and Social Care.

Competing interests None declared.

Patient consent for publication Not required.

Provenance and peer review Not commissioned; externally peer reviewed.

Supplemental material This content has been supplied by the author(s). It has not been vetted by BMJ Publishing Group Limited (BMJ) and may not have been peer-reviewed. Any opinions or recommendations discussed are solely those of the author(s) and are not endorsed by BMJ. BMJ disclaims all liability and responsibility arising from any reliance placed on the content. Where the content includes any translated material, BMJ does not warrant the accuracy and reliability of the translations (including but not limited to local regulations, clinical guidelines, terminology, drug names and drug dosages), and is not responsible for any error and/or omissions arising from translation and adaptation or otherwise.

Open access This is an open access article distributed in accordance with the Creative Commons Attribution Non Commercial (CC BY-NC 4.0) license, which permits others to distribute, remix, adapt, build upon this work non-commercially, and license their derivative works on different terms, provided the original work is properly cited, appropriate credit is given, any changes made indicated, and the use is non-commercial. See: http://creativecommons.org/licenses/by-nc/4.0/.

ORCID iD

Veronica Ranieri http://orcid.org/0000-0003-0528-8640

\section{REFERENCES}

1 Phelan AL, Katz R, Gostin LO. The novel coronavirus originating in Wuhan, China: challenges for global health governance. JAMA 2020;323:709-10.

2 Zhou F, Yu T, Du R, et al. Clinical course and risk factors for mortality of adult inpatients with COVID-19 in Wuhan, China: a retrospective cohort study. Lancet 2020;395:1054-62.

3 Wuhan Municipal Health Commission. Report of clustering pneumonia of unknown etiology in Wuhan City. Wuhan municipal health Commission, 2019. Wuhan: Wuhan Municipal Health Commission, 2019.

4 World Health Organisation. Coronavirus disease (COVID-19) advice for the public. Geneva: World Health Organisation, 2020.

5 Brooks SK, Webster RK, Smith LE, et al. The psychological impact of quarantine and how to reduce it: rapid review of the evidence. Lancet 2020;395:912-20.

6 Mihashi M, Otsubo Y, Yinjuan X, et al. Predictive factors of psychological disorder development during recovery following SARS outbreak. Health Psychol 2009;28:91-100.

7 Hawryluck L, Gold WL, Robinson S, et al. SARS control and psychological effects of quarantine, Toronto, Canada. Emerg Infect Dis 2004;10:1206-12.

8 Lee S, Chan LYY, Chau AMY, et al. The experience of SARS-related stigma at Amoy gardens. Soc Sci Med 2005;61:2038-46.

9 Reynolds DL, Garay JR, Deamond SL, et al. Understanding, compliance and psychological impact of the SARS quarantine experience. Epidemiol Infect 2008;136:997-1007.

10 Matsuishi K, Kawazoe A, Imai H, et al. Psychological impact of the pandemic (H1N1) 2009 on General Hospital workers in Kobe. Psychiatry Clin Neurosci 2012;66:353-60.

11 Shi L, Lu Z-A, Que J-Y, et al. Prevalence of and risk factors associated with mental health symptoms among the general population in China during the coronavirus disease 2019 pandemic. JAMA Netw Open 2020;3:e2014053.

12 Montemurro N. The emotional impact of COVID-19: from medical staff to common people. Brain Behav Immun 2020;87:23-4.

13 Serafini G, Parmigiani B, Amerio A, et al. The psychological impact of COVID-19 on the mental health in the general population. QJM 2020;113:531-7. doi:10.1093/qjmed/hcaa201

14 Gunnell D, Appleby L, Arensman E, et al. Suicide risk and prevention during the COVID-19 pandemic. Lancet Psychiatry 2020;7:468-71. 
15 Niederkrotenthaler T, Gunnell D, Arensman E, et al. Suicide research, prevention, and COVID-19. Crisis 2020;41:321-30.

16 Reger MA, Stanley IH, Joiner TE. Suicide mortality and coronavirus disease 2019-A perfect storm? JAMA Psychiatry 2020. doi:10.1001/jamapsychiatry.2020.1060. [Epub ahead of print: 10 Apr 2020].

17 Sher L. The impact of the COVID-19 pandemic on suicide rates. QJM 2020;113:707-12.

18 Costanza A, Amerio A, Odone A, et al. Suicide prevention from a public health perspective. what makes life meaningful? the opinion of some suicidal patients. Acta Biomed 2020;91:128-34.

19 Costanza A, Baertschi M, Richard-Lepouriel H, et al. Demoralization and its relationship with depression and Hopelessness in suicidal patients attending an emergency department. Int $J$ Environ Res Public Health 2020;17:2232.

20 Que J, Shi L, Deng J, et al. Psychological impact of the COVID-19 pandemic on healthcare workers: a cross-sectional study in China. Gen Psychiatr 2020;33:e100259.

21 Kang L, Ma S, Chen M, et al. Impact on mental health and perceptions of psychological care among medical and nursing staff in Wuhan during the 2019 novel coronavirus disease outbreak: a cross-sectional study. Brain Behav Immun 2020;87:11-17.

22 Amerio A, Bianchi D, Santi F, et al. Covid-19 pandemic impact on mental health: a web-based cross-sectional survey on a sample of Italian general practitioners. Acta Biomed 2020;91:83-8.

23 Stuijfzand S, Deforges C, Sandoz V, et al. Psychological impact of an epidemic/pandemic on the mental health of healthcare professionals: a rapid review. BMC Public Health 2020;20:1230.

24 Gardner W, Hoge SK, Bennett N, et al. Two scales for measuring patients' perceptions for coercion during mental hospital admission. Behav Sci Law 1993;11:307-21.

25 Hiday VA, Swartz MS, Swanson J, et al. Patient perceptions of coercion in mental hospital admission. Int J Law Psychiatry 1997;20:227-41.

26 Lidz CW, Mulvey EP, Hoge SK, et al. Factual sources of psychiatric patients' perceptions of coercion in the hospital admission process. Am J Psychiatry 1998;155:1254-60.
27 Amerio A, Brambilla A, Morganti A, et al. COVID-19 Lockdown: Housing Built Environment's Effects on Mental Health. Int J Environ Res Public Health 2020;17:5973.

28 Priebe S, Katsakou C, Yeeles K, et al. Predictors of clinical and social outcomes following involuntary hospital admission: a prospective observational study. Eur Arch Psychiatry Clin Neurosci 2011;261:377-86.

29 Lazarus RS, Folkman S. Stress, appraisal, and coping. New York: Springer, 1984.

30 Martínez-Mesa J, González-Chica DA, Bastos JL, et al. Sample size: how many participants do I need in my research? An Bras Dermatol 2014;89:609-15.

31 Maxwell SE. Sample size and multiple regression analysis. Psychol Methods 2000;5:434-58.

32 Moodle HQ. Moodle [program]. West Perth, Australia: Moodle HQ, 2018.

33 Blackboard Inc. Blackboard [program]. Washington DC: Blackboard Inc, 2018.

34 Peacock S, Robertson A, Williams S, et al. The role of learning technologists in supporting e-research. ALT-J 2009;17:115-29.

35 Lovibond SH, Lovibond PF. Manual for the Depression Anxiety \& Stress Scales. 2nd Ed. Sydney: Psychology Foundation, 1995.

36 Tedeschi RG, Calhoun LG. The posttraumatic growth inventory: measuring the positive legacy of trauma. J Trauma Stress 1996;9:455-71.

37 Carver CS. You want to measure coping but your protocol's too long: consider the brief cope. Int J Behav Med 1997;4:92-100.

38 Chong M-Y, Wang W-C, Hsieh W-C, et al. Psychological impact of severe acute respiratory syndrome on health workers in a tertiary hospital. Br J Psychiatry 2004;185:127-33.

39 Hudnall Stamm B. Professional quality of life: compassion satisfaction and fatigue version 5 (ProQOL), 2009. Available: www. proqol.org

40 QSR International. NVivo [program]. Melbourne, Australia: QSR International, 2018.

41 Braun V, Clarke V. Using thematic analysis in psychology. Qual Res Psychol 2006;3:77-101. 